\title{
Greed Supports Economic Growth But Might Make Us More Miserable
}

\author{
Ben David Nissim¹, Tavor Tchai ${ }^{1}$, Winer $\mathrm{Zvi}^{2}$ \\ ${ }^{1}$ Department of Economics and Management, Max Stern Academic College of Emek Yezreel, Jezreel Valley, \\ Israel \\ ${ }^{2}$ Department of Economics, Western Galilee Academic College, Akko, Israel \\ Email: nissimb@yvc.ac.il, ttavor@gmail.com,ZvikaV@wgalil.ac.il
}

Received 7 April 2016; accepted 6 June 2016; published 9 June 2016

Copyright (C) 2016 by authors and Scientific Research Publishing Inc.

This work is licensed under the Creative Commons Attribution International License (CC BY).

http://creativecommons.org/licenses/by/4.0/

(c) (i) Open Access

\begin{abstract}
Most economists, who refer to utility as representing wellbeing, do so under the assumption that utility increases with consumption. In contrast, lately researchers have found evidence that individuals' wellbeing is by far a more complicated matter than to be represented solely by their consumption choices. Adopting a broader approach to human wellbeing, we have modified the traditional theory to include income aspirations. Following this new line of thinking, this paper assumes that individuals seek to minimize the gap between their consumption aspirations and their consumption desires, namely minimizing their frustration. We present an overlapping generation model and assume that desires increase with current and lag consumption. Our theoretical results show that in an economy with agents minimizing frustration, as greed increases, the steady state level of capital might be higher while people would certainly be more miserable.
\end{abstract}

\section{Keywords}

Frustration, Desires, Equilibrium, Two Period Consumption Model

\section{Introduction}

Utility maximization is assumed to be the ultimate target of individuals. Almost all economic models that try to maximize the wellbeing of an individual or a group of individuals, assume that each one of them is trying to maximize utility from consumption. This school of thought is based on long living ideas starting with Hicks [1] and Allen [2]. The dominant line of thinking in standard economic theory, which is based on observable choices made by individuals, assumes that individual utility depends on tangible goods, services and leisure. Individuals' behavior reveals their preferences which explain their choices. 
Surveying the literature regarding human happiness and the connection between wealth and happiness reveals that most economists who make pronouncements about economic matters do so under a set of assumptions about human happiness. Chief of these is the belief that by raising income or output an individual is better off.

Significant deviation from this paradigm has been shown by Pigou [3]. He argued that appetite might grow with eating, making desire more intense. Thus, growing consumption has two affects, the first by itself and the second through growing desire. The second affect may be so high that more consumption necessarily implying more satisfaction or happiness is not the case. This is because unsatisfied desire is usually painful. "If a man with a given income of food per day becomes hungrier, the utility associated with the food he has increases, but the disutility of the food he has not increases too; and the last state of that man may be worse than the first". Compatible with Pigous' argumentation [3], Barnes [4] showed that across certain populations suicide rates had a positive correlation with income.

In the years after Pigou argued his revolutionary claim, economic theory has dealt with the issue of the relationship between income and happiness. Easterlin [5] asked the crucial question "Are the wealthy members of society usually happier than the poor?". By using statistical data, he has showed that individual happiness is not different in poor and rich countries and that economic growth does not necessarily bring happiness. He argued that happiness was determined by comparison of individuals to their environment. In a later paper, Easterlin [6], brought evidence from time series studies of the United States, nine European countries, and Japan that within a country, at a given time, those with higher income are on average happier, but, raising the income of all does not increase the happiness of all. The reason is that the norms and standards on which the individual judgments of happiness are based increase in the same proportion as income increases. He stated that individuals were allowed to define their own standards of happiness and support the approach, started by Cantril [7], that rising income changes individuals' tastes, causing them to have ever-increasing aspirations. Supporting this argument, Hirsch [8] and Scitovsky [9] showed that individuals' happiness did not rise when economic growth was an overall trend so that relatively no one stood out. Frey and Stutzer [10] raised the possibility that additional income does not necessarily raise happiness.

Recognizing that economic variables are not what interest the public, but rather the happiness derived from them, Oswald [11] stated that economic indicators are of interest to the public. These metrics are merely a means to bring happiness. "The relevance of economic performance is that it may be a means to an end. That end is not the consumption of beef burgers, nor the accumulation of television sets, nor the vanquishing of some high level of interest rates, but rather the enrichment of mankind's feeling of wellbeing. Economic things matter only in so far as they make people happier". Leightner (2005) also challenged the thought that utility is the ultimate objective of individuals. He acknowledged the fact that utility is a mean to achieve a goal and not a goal itself.

Further deviation from the traditional utility theory was made by Rabin [12]. He brought overwhelming evidence that people are often more sensitive to how their current situation differs from some reference level than to the absolute characteristics of the situation. For instance, a level of income that seems low to us if we belong to a group with a high income level seems high to us if we belong to a group with a low income level. Given this finding, we ought to incorporate habitual levels of consumption into individuals' utility factors. Therefore, past consumption or expectations of future consumption are no less important than current consumption. In a seminal work, Stutzer [13] raised the important question of what the role of individual aspirations is in determining one's utility, aside from income and consumption level. Empirical data show that, ceteris paribus, higher aspirations reduce utility. "Two processes are theoretically put forward as forming individual aspirations. First, there is individuals' adaptation to repeated stimuli, as provided by people's consumption habits. Whereas additional material goods and services initially provide extra pleasure, their effects wear off over time. Thus people get used to their consumption and income level. Second, there are social comparisons with relevant to others. It is not the absolute level of income that matters most, but rather one's position relative to other individuals". This argument has been reinforced by Luttmer [14] who showed a data panel proving that individuals feel worse off when others around them earn more.

Finally, significant strengthen regarding the importance of happiness rather than utility comes from Kahneman and Krueger [15] who argued that "For good reasons, economists have had a long-standing preference for studying peoples' revealed preferences; that is, looking at individuals' actual choices and decisions rather than their stated intentions or subjective reports of likes and dislikes. Yet people often make choices that bear a mixed relationship to their own happiness". 
As we can see, the main points raised in the literature review are that an increase in income does not necessarily increases individuals' utility or happiness. Past consumption, future consumption and aspirations play a major role in determining individuals' happiness. Although growing income and consumption raise utility, it may raise the desire for even more and therefore create a growing and painful gap, causing disutility.

Based on Pigou [3], Rabin [12] Frey and Stutzer [10], Stutzer [13], and Luttmer [14] among others, we assume in this paper that individuals have desires to consume that increase with the current and lag level of consumption. As the gap between desires and consumption is higher, an individual will be less happy (more miserable). Under such conditions, the ultimate goal of individuals is to minimize misery during their life time.

\section{Model Structure}

Consider a perfectly competitive world where economic activity is performed over infinite discrete time, $t=1,2, \cdots, \infty$.

All individuals live during two periods of time. An individual works during the first period and retires at the beginning of the second. Each individual saves part of his first period income so that the savings, including the return, finance his second period consumption. During each period, both young and old people are alive. The rate of population growth equals zero.

\subsection{Consumption and Savings}

In every period, $t$, a generation which consists of $L_{t}$ individuals, is born.

During the first period, they work and earn a certain wage, and during the second period they are retired. Individuals, $i$, born at time $t$, are characterized by their intertemporal pleasure function $U\left(C_{0 t}^{i}, C_{1 t}^{i}\right)$ defined over non-negative consumption during the first and second periods of their live, Where $C_{0 t}^{i}$ is the consumption of individual $i$, who was born at period $\mathrm{t}$, when he is young and $C_{1 t}^{i}$ is the consumption of individual $i$, who was born at period $t$, when he is old.

Each individual has desires he tries to fulfill. Let us define $D\left(C_{0 t}^{i}, C_{1 t}^{i}\right)$ as the inter-temporal desire level. Assuming that desire is always higher than pleasure, each agent will try to maximize the negative term $U\left(C_{0 t}^{i}, C_{1 t}^{i}\right)-D\left(C_{0 t}^{i}, C_{1 t}^{i}\right)$.

During the first period of their lifetime, individuals born at time $t$ supply their unit endowment of labor inelastically. The resulting wage income is allocated between first period consumption $C_{0 t}^{i}$ and savings $S_{t}^{i}$.

Each individual tries to maximize his utility subject to his two period budget constraint:

$$
\begin{array}{ll}
\text { Min } & U\left(C_{0 t}^{i}, C_{1 t}^{i}\right)-D\left(C_{0 t}^{i}, C_{1 t}^{i}\right) \\
\text { s.t } & C_{0 t}^{i}+\frac{C_{1 t}^{i}}{1+r}=W_{0 t}^{i}(1-t)+\frac{W_{1 t}^{i}(1-t)}{1+r}
\end{array}
$$

$W_{0 t}^{i}$ is the wage of individual $i$, born at period $t$, when he is young.

$W_{1 t}^{i}$ is the wage of individual $i$, born at period $t$, when he is old.

$t$ is the tax rate.

$W_{1 t}^{i}=0$, since he doesn't work when old.

For simplicity's sake, we can define $W_{0 t}^{i}=W_{i t}$.

(2.1.1) Specific Utility

Let us assume that the pleasure function of each agent in the economy is: $U=\ln \left(C_{0 t}\right)+\gamma \ln \left(C_{1 t}\right)$.

While desire function is formulated according to the assumption that the level of desire increases with consumption, which relies on the thought that rising income changes an individual's tastes causing him to have ever-increasing aspirations (see Cantril [7], Rabin [12] and Pigou [3]).

According to this way of thinking, we assume that in each period desire increases with current and with lag consumption, and define it as:

$$
D=\ln \left(C_{0}^{\eta}\right)+\lambda \ln \left(C_{1 t}^{\delta}\right)+\lambda \ln \left(C_{0}^{\psi}\right)=\eta \ln \left(C_{0}\right)+\lambda \delta \ln \left(C_{1 t}\right)+\lambda \psi \ln \left(C_{0}\right), \delta>1, \eta>1 .
$$


where:

$\gamma<1$ is the discount factor of utility in period 1 .

$\lambda<1$ is the discount factor of utility in the desire function-D at period 1 .

$\eta>1$ is a factor that increases desires in period 0 as consumption in period 0 is higher.

$\delta>1$ is a factor that increases desires in period 1 as consumption in period 1 is higher.

$\psi>1$ is a factor that increases desires in period 1 as consumption in period 0 is higher.

Notice that discounted desire level in the second period increases with consumption in period 0 and 1 (according to term $\lambda \ln \left(C_{1 t}^{\delta}\right)+\lambda \ln \left(C_{0}^{\psi}\right)$ the memory of the amount consumed in period 0 , as well as the current consumption increases desire in period 1 ).

The agent is trying to maximize the term:

$$
\begin{aligned}
U-D & =-\eta \ln \left(C_{0}\right)-\lambda \delta \ln \left(C_{1 t}\right)-\lambda \psi \ln \left(C_{0}\right)+\left[\ln \left(C_{0 t}\right)+\gamma \ln \left(C_{1 t}\right)\right] \\
& =(1-\eta-\lambda \psi) \ln \left(C_{0 t}\right)+(\gamma-\lambda \delta) \ln \left(C_{1 t}\right)
\end{aligned}
$$

subject to his budget constraints, as presented in Equation (1b).

$$
\begin{array}{ll}
\text { MAX } & (1-\eta-\lambda \psi) \ln \left(C_{0 t}\right)+(\gamma-\lambda \delta) \ln \left(C_{1 t}\right) \\
\text { s.t } & C_{0 t}^{i}+\frac{C_{1 t}^{i}}{1+r}=W_{i t}(1-t)
\end{array}
$$

where, $t$ represents the tax rate.

We get:

$$
\begin{aligned}
C_{0 t} & =\frac{(\eta+\lambda \psi-1)}{(\eta+\lambda \psi-1)+(\lambda \delta-\gamma)} \cdot W t(1-t) \\
C_{1 t} & =\frac{(\lambda \delta-\gamma)(1+r)}{(\eta+\lambda \psi-1)+(\lambda \delta-\gamma)} \cdot W t(1-t) \\
S_{t} & =\frac{(\lambda \delta-\gamma)}{(\eta+\lambda \psi-1)+(\lambda \delta-\gamma)} \cdot W t(1-t)
\end{aligned}
$$

$S_{t}$ is the amount saved in period 0.

\subsection{Production, Income Distribution and Savings}

For simplicity's sake, let us assume that there is one active firm in the market in period $t$.

$L_{t}$ represents the number of workers in period $t$.

The output produced by the firm at time $t$ is:

$$
Y_{t}=K_{t}^{\alpha} L_{t}^{\beta}
$$

$K_{t}$ is the amount of capital.

(2.2.1) Income distribution

The product, $Y_{t}$, in the firm is divided between the firm owners and the workers. If labor is perfectly mobile, then workers get their marginal product, so that:

$$
W_{t}=\beta K_{t}^{\alpha} L_{1 t}^{(\beta-1)}
$$

where $W_{t}$ is the wage of a worker.

In each firm we get that: $W_{t} L_{t}=\left(\beta K^{\alpha} L_{t}^{(\beta-1)}\right) L_{t}=\beta Y_{t}$ is the total income of the workers.

The income of the firm owners is: $W_{p, t}=K_{t}^{\alpha} L_{t}^{\beta}-\left(\beta K^{\alpha} L_{t}^{(\beta-1)}\right) L_{t}=(1-\beta) Y_{t}$.

(2.2.2) Aggregate savings

Assuming that the number of workers is $\mathrm{N}$ and that there is 1 firm owner, the aggregate savings in the economy would be: 


$$
S_{t}=\sum_{i=1}^{N+1} S_{t}^{i}=\frac{\lambda \delta-\gamma}{(\eta+\lambda \psi-1)+(\lambda \delta-\gamma)} \sum_{i=1}^{N+1} W_{t}^{i}(1-t)=\frac{\lambda \delta-\gamma}{(\eta+\lambda \psi-1)+(\lambda \delta-\gamma)} Y_{t}(1-t)
$$

Since $\lambda$ and $\gamma$, the discount factors of utility in period 1 , are smaller than 1 while the factor that increase desires $\delta, \eta$ and $\psi$ are much larger than 1 (we suppose that desires grow exponentially with current and past levels of consumption), $\lambda \delta-\gamma$ and $\eta+\lambda \psi-1>0$.

\subsection{Equilibrium}

The amount of capital in the next generation would be $K_{t+1}=(1-d) K_{t}+I_{t}$ and the net investment would be financed by the savings of the current generation, so that $S_{t}=I_{t}$. We get that:

$$
K_{t+1}=(1-d) K_{t}+S_{t}
$$

Substituting (4) in (5) we get:

$$
K_{t+1}=(1-d) K_{t}+\frac{\lambda \delta-\gamma}{(\eta+\lambda \psi-1)+(\lambda \delta-\gamma)} Y_{t}(1-t)
$$

Substituting the production function (2) into (6) we get:

$$
K_{t+1}=(1-d) K_{t}+\frac{\lambda \delta-\gamma}{(\eta+\lambda \psi-1)+(\lambda \delta-\gamma)} K_{t}^{\alpha} L_{t}^{\beta}(1-t)
$$

Given that population size is fixed, we get that the capital stock of the next generation is determined by the current stock of capital.

Stationary equilibrium will exist for:

$$
K_{t+1}=K_{t}
$$

Substituting (7) into (6a) we get:

$$
K_{t}=(1-d) K_{t}+\frac{\lambda \delta-\gamma}{(\eta+\lambda \psi-1)+(\lambda \delta-\gamma)} K_{t}^{\alpha} L_{t}^{\beta}(1-t)
$$

or

$$
K_{t}=\left[\frac{\lambda \delta-\gamma}{(\eta+\lambda \psi-1)+(\lambda \delta-\gamma)} \cdot \frac{L_{t}^{\beta}(1-t)}{d}\right]^{1 / 1-\alpha}
$$

For $K_{t}$ the stationary amount of capital.

Substituting ( $7 b)$ into (2) we get the stationary amount of production is:

$$
Y_{t}=\left[\frac{\lambda \delta-\gamma}{(\eta+\lambda \psi-1)+(\lambda \delta-\gamma)} \cdot \frac{L_{t}^{\beta}(1-t)}{d}\right]^{\alpha / 1-\alpha} \cdot L_{t}^{\beta}
$$

or

$$
Y_{t}=\left[\frac{\lambda \delta-\gamma}{(\eta+\lambda \psi-1)+(\lambda \delta-\gamma)} \cdot \frac{1-t}{d}\right]^{\alpha / 1-\alpha} \cdot L_{t}^{\beta / 1-\alpha}
$$

Substituting (7b) into (3) we get the stationary wage of each worker is:

$$
W_{t}=\beta \cdot\left[\frac{\lambda \delta-\gamma}{(\eta+\lambda \psi-1)+(\lambda \delta-\gamma)} \cdot \frac{L_{t}^{\beta}(1-t)}{d}\right]^{\alpha / 1-\alpha} \cdot L_{t}^{\beta}
$$

Substituting (9) into Equations (1c) and (1d) we get the stationary level of consumption in period 0 and 1 are:

$$
C_{0}=\frac{(\eta+\lambda \psi-1)}{(\eta+\lambda \psi-1)+(\lambda \delta-\gamma)} \cdot\left[\frac{\lambda \delta-\gamma}{(\eta+\lambda \psi-1)+(\lambda \delta-\gamma)} \cdot \frac{L^{\beta}(1-t)}{d}\right]^{\alpha / 1-\alpha} \cdot \beta L^{\beta}(1-t)
$$


or

$$
C_{0}=\frac{(\eta+\lambda \psi-1)(\lambda \delta-\gamma)^{\alpha / 1-\alpha}}{[(\eta+\lambda \psi-1)+(\lambda \delta-\gamma)]^{1 / 1-\alpha}} \cdot\left[\frac{L^{\beta}(1-t)}{d}\right]^{\alpha / 1-\alpha} \cdot \beta L^{\beta}(1-t)
$$

and

$$
C_{1}=\frac{(\lambda \delta-\gamma)(1+r)}{(\eta+\lambda \psi-1)+(\lambda \delta-\gamma)} \cdot\left[\frac{\lambda \delta-\gamma}{(\eta+\lambda \psi-1)+(\lambda \delta-\gamma)} \cdot \frac{L^{\beta}(1-t)}{d}\right]^{\alpha / 1-\alpha} \cdot \beta L^{\beta}(1-t)
$$

or

$$
C_{1}=\frac{(\lambda \delta-\gamma)^{1 / 1-\alpha}(1+r)}{[(\eta+\lambda \psi-1)+(\lambda \delta-\gamma)]^{1 / 1-\alpha}} \cdot\left[\frac{L^{\beta}(1-t)}{d}\right]^{\alpha / 1-\alpha} \cdot \beta L^{\beta}(1-t)
$$

While substituting (8) into (4) we get the stationary level of total saving is:

$$
S=\frac{(\lambda \delta-\gamma)}{(\eta+\lambda \psi-1)+(\lambda \delta-\gamma)} \cdot\left[\frac{\lambda \delta-\gamma}{(\eta+\lambda \psi-1)+(\lambda \delta-\gamma)} \cdot \frac{L^{\beta}(1-t)}{d}\right]^{\alpha / 1-\alpha} \cdot L^{\beta}(1-t)
$$

or:

$$
S=\frac{(\lambda \delta-\gamma)^{1 / 1-\alpha}}{[(\eta+\lambda \psi-1)+(\lambda \delta-\gamma)]^{1 / 1-\alpha}} \cdot\left[\frac{L^{\beta}(1-t)}{d}\right]^{\alpha / 1-\alpha} \cdot L^{\beta}(1-t)
$$

Minimum frustration in steady state (2.3.1)

Substituting (10a) and (11a) into (1a) we get that frustration level in steady state is:

$$
\begin{aligned}
U-D= & (1-\eta-\lambda \psi) \cdot \ln \left\{\frac{(\eta+\lambda \psi-1)(\lambda \delta-\gamma)^{\alpha / 1-\alpha}}{[(\eta+\lambda \psi-1)+(\lambda \delta-\gamma)]^{1 / 1-\alpha}} \cdot\left[\frac{L^{\beta}(1-t)}{d}\right]^{\alpha / 1-\alpha} \cdot \beta L^{\beta}(1-t)\right\} \\
& +(\gamma-\lambda \delta) \cdot \ln \left\{\frac{(\lambda \delta-\gamma)^{1 / 1-\alpha}(1+r)}{[(\eta+\lambda \psi-1)+(\lambda \delta-\gamma)]^{1 / 1-\alpha}} \cdot\left[\frac{L^{\beta}(1-t)}{d}\right]^{\alpha / 1-\alpha} \cdot \beta L^{\beta}(1-t)\right\}
\end{aligned}
$$

\subsection{Comparative Statics}

We can present (13) as:

$$
\begin{aligned}
U-D= & -[(\eta+\lambda \psi-1)+(\lambda \delta-\gamma)] \ln \left\{\left[\frac{\lambda \delta-\gamma}{(\eta+\lambda \psi-1)+(\lambda \delta-\gamma)}\right]^{1 / 1-\alpha} \cdot\left[\frac{L^{\beta}(1-t)}{d}\right]^{\alpha / 1-\alpha} \cdot \beta L^{\beta}(1-t)\right\} \\
& -(\eta+\lambda \psi-1) \cdot \ln \left[\frac{\eta+\lambda \psi-1}{\lambda \delta-\gamma}\right]-(\lambda \delta-\gamma) \cdot \ln (1+r)
\end{aligned}
$$

We concentrate at the effect of the greed parameters $\eta, \psi$ and $\delta$ on the amount of capital, output and on frustration level in steady state.

2.4.1. The Effect of $\eta, \psi$ and $\delta$ on Capital and Output Differentiating (7b) and (8a) with respect to $\eta$ we get:

$$
\frac{\partial K}{\partial \eta}=\left[\frac{(\lambda \delta-\gamma) L^{\beta}(1-t)}{d}\right]^{1 / 1-\alpha} \cdot \frac{1}{1-\alpha} \cdot \frac{-1}{[(\eta+\lambda \psi-1)+(\lambda \delta-\gamma)]^{2-\alpha / 1-\alpha}}<0
$$




$$
\frac{\partial Y}{\partial \eta}=\left[\frac{(\lambda \delta-\gamma) L^{\beta}(1-t)}{d}\right]^{\alpha / 1-\alpha} \cdot \frac{\alpha L^{\beta}}{1-\alpha} \cdot \frac{-1}{[(\eta+\lambda \psi-1)+(\lambda \delta-\gamma)]^{2-\alpha / 1-\alpha}}<0
$$

As we explained above, since $\lambda \delta-\gamma>0$ and $(\eta+\lambda \psi-1)>0$, all the expressions in (14) and in (15) are smaller than zero.

Differentiating (7b) and (8a) with respect to $\psi$ we get:

$$
\begin{gathered}
\frac{\partial K}{\partial \psi}=\left[\frac{(\lambda \delta-\gamma) L^{\beta}(1-t)}{d}\right]^{1 / 1-\alpha} \cdot \frac{1}{1-\alpha} \cdot \frac{-\lambda}{[(\eta+\lambda \psi-1)+(\lambda \delta-\gamma)]^{2-\alpha / 1-\alpha}}<0 \\
\frac{\partial Y}{\partial \psi}=\left[\frac{(\lambda \delta-\gamma) L^{\beta}(1-t)}{d}\right]^{\alpha / 1-\alpha} \cdot \frac{\alpha L^{\beta}}{1-\alpha} \cdot \frac{-\lambda}{[(\eta+\lambda \psi-1)+(\lambda \delta-\gamma)]^{2-\alpha / 1-\alpha}}<0
\end{gathered}
$$

As we can see, the same analysis regarding (14) and (15) applies here and the expressions in (16) and in (17) are smaller than zero.

Differentiating (7b) and (8a) with respect to $\delta$ we get:

$$
\begin{aligned}
& \frac{\partial K}{\partial \delta}=\left[\frac{L^{\beta}(1-t)}{d}\right]^{1 / 1-\alpha} \cdot \frac{1}{1-\alpha} \cdot\left[\frac{\lambda \delta-\gamma}{(\eta+\lambda \psi-1)+(\lambda \delta-\gamma)}\right]^{\alpha / 1-\alpha} \cdot \frac{\lambda(\eta+\lambda \psi-1)}{[(\eta+\lambda \psi-1)+(\lambda \delta-\gamma)]^{2}}>0 \\
& \frac{\partial Y}{\partial \delta}=\left[\frac{L^{\beta}(1-t)}{d}\right]^{\alpha / 1-\alpha} \cdot \frac{\alpha L^{\beta}}{1-\alpha} \cdot\left[\frac{\lambda \delta-\gamma}{(\eta+\lambda \psi-1)+(\lambda \delta-\gamma)}\right]^{2 \alpha-1 / 1-\alpha} \cdot \frac{\lambda(\eta+\lambda \psi-1)}{[(\eta+\lambda \psi-1)+(\lambda \delta-\gamma)]^{2}}>0
\end{aligned}
$$

As we can see for $\lambda \delta-\gamma>0$ and $(\eta+\lambda \psi-1)>0$ the expressions in (18) and in (19) are larger than zero.

According to the comparative statics in (14), (16) and (18) as greed increases in the first period due to current consumption, and as greed increases in the second period due to consumption memory of the first period, capital and output levels in steady state would be lower. However, as greed increases in the second period due to the consumption level in the second period, capital stock in steady state will be higher.

In other words, economic growth is negatively affected by greed originated by rising consumption in the first period and is positively affected by greed originated by rising consumption in the second period.

\subsubsection{The Effect of $\eta, \psi$ and $\delta$ on Frustration (Misery)}

Differentiating (13a) with respect to $\eta$ we get:

$$
\frac{\partial(U-D)}{\partial \eta}=-\ln \left\{\left[\frac{\lambda \delta-\gamma}{(\eta+\lambda \psi-1)+(\lambda \delta-\gamma)}\right]^{1 / 1-\alpha} \cdot\left[\frac{L^{\beta}(1-t)}{d}\right]^{\alpha / 1-\alpha} \cdot \beta L^{\beta}(1-t)\right\}+\frac{\alpha}{1-\alpha}-\ln \left[\frac{\eta+\lambda \psi-1}{\lambda \delta-\gamma}\right]<0
$$

See more details on differentiation in (20) in Appendix 1.

As we can see, all the terms in (20) are smaller than zero, except the term $\frac{\alpha}{1-\alpha}$, which is positive (given that $\alpha$, is at a size of about 0.25 , this term is equal to 0.333 ). Notice that the other expressions in (20) are probably much smaller since $L$, the number of workers, is very large and the terms $(\eta+\lambda \psi-1)$ and $\lambda \delta-\gamma$ are positive. (Equation (20) can be presented as $\frac{\partial(U-D)}{\partial \eta}=-\ln \left(\frac{C_{1}}{1+r}\right)+\frac{\alpha}{1-\alpha}-\ln \left[\frac{\eta+\lambda \psi-1}{\lambda \delta-\gamma}\right]<0$. Notice that $\ln (1.4)=0.33$, and the negative terms $-\ln \left(\frac{C_{1}}{1+r}\right)$ are probably much larger than 0.33 in absolute value).

Differentiating (13a) with respect to $\psi$ we get: 


$$
\begin{aligned}
\frac{\partial(U-D)}{\partial \psi}= & -\lambda \cdot \ln \left\{\left[\frac{\lambda \delta-\gamma}{(\eta+\lambda \psi-1)+(\lambda \delta-\gamma)}\right]^{1 / 1-\alpha} \cdot\left[\frac{L^{\beta}(1-t)}{d}\right]^{\alpha / 1-\alpha} \cdot \beta L^{\beta}(1-t)\right\} \\
& +\frac{\lambda \alpha}{1-\alpha}-\ln \left[\frac{\eta+\lambda \psi-1}{\lambda \delta-\gamma}\right]<0
\end{aligned}
$$

As we can see, all the terms are smaller than zero, except the term $\frac{\lambda \alpha}{1-\alpha}$ which is positive at a size of smaller than 0.333 . The same analysis regarding (20) applies here and all the expressions in (21) are smaller than zero.

Differentiating (13a) with respect to $\delta$ we get:

$$
\begin{aligned}
\frac{\partial(U-D)}{\partial \delta}= & -\lambda \cdot \ln \left\{\left[\frac{\lambda \delta-\gamma}{(\eta+\lambda \psi-1)+(\lambda \delta-\gamma)}\right]^{1 / 1-\alpha} \cdot\left[\frac{L^{\beta}(1-t)}{d}\right]^{\alpha / 1-\alpha} \cdot \beta L^{\beta}(1-t)\right\} \\
& -\frac{\alpha \lambda(\eta+\lambda \psi-1)}{(1-\alpha)(\lambda \delta-\gamma)}-\lambda \cdot \ln (1+r)<0
\end{aligned}
$$

The analysis regarding (20) applies here as well and the expressions in (22) are smaller than zero.

As we can see, increased greed would make people less happy (Table 1).

\section{How Is Economic Growth Changed with Greed?}

In this section, we compared steady state levels of capital in two models. The first is a standard overlapping generation model with individuals that maximize utility while the second is an overlapping generation model with individuals that minimize frustration.

Appendix 2 presents the results for the first model. We get that steady state levels of capital and production are:

$$
\begin{gathered}
K_{t}=\left[\frac{\gamma}{1+\gamma} \cdot \frac{L_{t}^{\beta}(1-t)}{d}\right]^{1 / 1-\alpha} \\
Y_{t}=\left[\frac{\gamma}{1+\gamma} \cdot \frac{1-t}{d}\right]^{\alpha / 1-\alpha} \cdot L_{t}^{\beta / 1-\alpha}
\end{gathered}
$$

Comparing steady state levels of capital and output in the first model (Equations (23) and (24)) to their steady state levels in the second model (Equations (7b) and (8a)), we can see that if:

$$
\frac{\lambda \delta-\gamma}{(\eta+\lambda \psi-1)+(\lambda \delta-\gamma)}>\frac{\gamma}{1+\gamma}
$$

then the steady state level of capital and production in the second model are higher.

After some manipulations, we get that Equation (25) is true if

$$
\gamma<\frac{\lambda(\delta-\psi \gamma)}{\eta}
$$

Table 1. Summarizing the results of the comparative statics.

\begin{tabular}{cccc}
\hline & $\delta$ & $\eta$ & $\psi$ \\
\hline$K$ & $\uparrow$ & $\uparrow$ & $\uparrow$ \\
$Y$ & $\uparrow$ & $\uparrow$ & $\uparrow$ \\
$U-D$ & $\downarrow$ & $\downarrow$ & $\downarrow$ \\
\hline
\end{tabular}


We can present (25a) as:

$$
\frac{(\lambda \delta)}{\eta+\lambda \psi}>\gamma
$$

Notice that $\gamma$ and $\lambda$ are discount factors of utility (smaller than 1 ).

We get that as $\psi$ and $\eta$, the factors that increase desires in period 0 and period 1 according to consumption in period 0 are higher, an economy with agents that takes into consideration the effect of greed will converge to a lower steady state level of capital and production. However as $\delta$, the factor that increases desires in period 1 according to consumption level in period 1 is higher, the economy will converge to a higher steady state level of capital and production.

These results are in the same direction pointed by the comparative statics (In the comparative statics, we showed that $\psi$ and $\eta$ have a negative affect while $\delta$ has a positive effect on steady state level of capital).

In order to interpret the results, let us assume the existence of one economy with agents that maximize utility and a second economy with agents that maximize the net utility (U-D). The second economy would reach higher steady state level of capital as agents become greedier due to higher consumption in the second period of their lives and as their greed increases in smaller magnitude due to their consumption in the first period of their lives.

In summary, in an economy with agents that take into consideration the frustration caused by unsatisfied desires, a higher steady state level of capital will be reached compared to a traditional economy with maximizing utility agents if the evolving greed is higher when people are old and lower when people are young.

\section{Conclusions}

Classic economic literature refers to a human agent as a homo-economicus who has one main aim, namely to maximize utility from consumption. According to this school of thought, all the economic decisions made by an agent are meant to reach the goal of maximizing utility. However, we find many researchers who find economic behaviors that do not meet this approach.

In this paper, we suggest a revised approach and assume that individuals are trying to maximize the net utility which we define as the difference between utility and desires (which is similar to an approach of trying to minimize misery). According to our model, the agents' desires are strengthened by current and past levels of consumption. Although this way of thinking is not new (see for example Cantril [7], Rabin [12], Pigou [3], Frey and Stutzer [10]) we could not find theoretical models that analyzed the connection between economic growth and human wellbeing while assuming that agents tried to minimize their misery.

We present an overlapping generation model with agents that have desires which increase with current and past consumption. We define misery (or frustration) as the difference between the level of utility and the level of desires. Each agent lives for two periods: working during the first and being retired during the second. The agent uses his first period income for consumption and for savings. During the second period, the agent consumes his savings plus interest earned. The aggregate amount saved in the first period finances investment which changes the amount of capital in the second period. As capital increases, production and income increase.

Steady state for the amount of capital, production and wage is calculated. Given steady state level of wage, the difference between utility and desire, which we defined as frustration is calculated in steady state.

The amounts of capital, production and frustration in steady state are defined as a function of the desire parameters. Using comparative statics, we find that as agents become greedier due to higher consumption in the second period of their lives and as their greed increases in smaller magnitude due to their consumption in the first period of their lives, steady state level of capital and production will be higher.

In order to examine the findings, we compared capital steady state level in our model to steady state of capital in a standard model with agents that maximized their utility, while using the same production function.

We find that an economy with agents minimizing frustration will reach higher steady state of level of capital compared to a traditional economy if the evolving greed due to consumption in the first period is smaller and the evolving greed due to consumption in the second period is higher.

According to our comparative statics, higher greed at any stage of life is accompanied by a reduction in agents' wellbeing.

The results indicate that in cases where people become greedier in the second period of their life, the economy will grow in a larger magnitude while people will be more miserable. 


\section{References}

[1] Hicks, J.R. (1934) A Reconsideration of the Theory of Value, I. Economica, 1, 52-75. http://dx.doi.org/10.2307/2548574

[2] Allen, R.G.D. (1934) A Reconsideration of the Theory of Value, II. Economica, 1, 196-219. http://dx.doi.org/10.2307/2548749

[3] Pigou, A.C. (1951) Some Aspects of Welfare Economics. American Economic Review, 41, 167, $287-302$.

[4] Barnes, C.B. (1975) The Partial Effect of Income on Suicide Is Always Negative. American Journal of Sociology, 80, 454-460. http://dx.doi.org/10.1086/226002

[5] Easterlin, R. (1974) Does Economic Growth Improve the Human Lot? Some Empirical Evidence. In: David, P.A. and Reder, M.W., Eds., Nations and Household in Economic Growth, Academic Press, New York, 89-125.

[6] Easterlin, R. (I995) Will Raising the Incomes of All Increase the Happiness of All? Journal of Economic Behaviour and Organization, 27, 35-48. http://dx.doi.org/10.1016/0167-2681(95)00003-B

[7] Cantril, H. (1965) The Pattern of Human Concerns. Rutgers University Press, New Brunswick.

[8] Hirsch, F. (1976) The Social Limits of Growth. Harvard University Press, Cambridge. http://dx.doi.org/10.4159/harvard.9780674497900

[9] Scitovsky, T. (1976) The Joyless Economy. Oxford University Press, Oxford.

[10] Frey, B.S. and Stutzer, A. (2002) What Can Economists Learn from Happiness Research? Journal of Economic Literature, 40, 402-435. http://dx.doi.org/10.1257/jel.40.2.402

[11] Oswald, A.J. (1997) Happiness and Economic Performance. Economic Journal, 107, 1815-1831. http://dx.doi.org/10.1111/j.1468-0297.1997.tb00085.x

[12] Rabin, M. (1998) Psychology and Economics. Journal of Economic Literature, 36, 11-46.

[13] Stutzer, A. (2004) The Role of Income Aspirations in Individual Happiness. Journal of Economic Behavior and Organization, 54, 89-109. http://dx.doi.org/10.1016/j.jebo.2003.04.003

[14] Luttmer, E.F.P. (2005) Neighbors as Negatives: Relative Earnings and Wellbeing. Quarterly Journal of Economics, 120, 963-1002.

[15] Kahneman, D. and Krueger, A.B. (2006) Developments in the Measurement of Subjective Well-Being. Journal of Economic Perspectives, 20, 3-24. http://dx.doi.org/10.1257/089533006776526030 


\section{Appendix 1}

\section{Comparative Statics}

1. Detailed differentiation with respect to $\eta$ :

$$
\begin{aligned}
\frac{\partial(U-D)}{\partial \eta}= & -\ln \left\{\left[\frac{\lambda \delta-\gamma}{(\eta+\lambda \psi-1)+(\lambda \delta-\gamma)}\right]^{1 / 1-\alpha} \cdot\left[\frac{L^{\beta}(1-t)}{d}\right]^{\alpha / 1-\alpha} \cdot \beta L^{\beta}(1-t)\right\} \\
& +\frac{\frac{1}{1-\alpha} \cdot[(\eta+\lambda \psi-1)+(\lambda \delta-\gamma)] \cdot\left[\frac{L^{\beta}(1-t)}{d}\right]^{\alpha / 1-\alpha} \cdot \beta L^{\beta}(1-t)}{\left\{\left[\frac{\lambda \delta-\gamma}{(\eta+\lambda \psi-1)+(\lambda \delta-\gamma)}\right]^{1 / 1-\alpha} \cdot\left[\frac{L^{\beta}(1-t)}{d}\right]^{\alpha / 1-\alpha} \cdot \beta L^{\beta}(1-t)\right\}} \\
& \cdot\left[\frac{\lambda \delta-\gamma}{(\eta+\lambda \psi-1)+(\lambda \delta-\gamma)}\right]^{\alpha / 1-\alpha} \cdot \frac{\lambda \delta-\gamma}{[(\eta+\lambda \psi-1)+(\lambda \delta-\gamma)]^{2}} \\
& -\ln \left[\frac{\eta+\lambda \psi-1}{\lambda \delta-\gamma}\right]-(\eta+\lambda \psi-1) \cdot \frac{1}{\frac{\eta+\lambda \psi-1}{\lambda \delta-\gamma} \cdot \frac{1}{\lambda \delta-\gamma}}
\end{aligned}
$$

2. Detailed differentiation with respect to $\psi$ :

$$
\begin{aligned}
\frac{\partial(U-D)}{\partial \psi}= & -\lambda \cdot \ln \left\{\left[\frac{\lambda \delta-\gamma}{(\eta+\lambda \psi-1)+(\lambda \delta-\gamma)}\right]^{1 / 1-\alpha} \cdot\left[\frac{L^{\beta}(1-t)}{d}\right]^{\alpha / 1-\alpha} \cdot \beta L^{\beta}(1-t)\right\} \\
& +\frac{1}{1-\alpha} \cdot[(\eta+\lambda \psi-1)+(\lambda \delta-\gamma)] \cdot\left[\frac{L^{\beta}(1-t)}{d}\right]^{\alpha / 1-\alpha} \cdot \beta L^{\beta}(1-t) \\
& \left\{\left[\frac{\lambda \delta-\gamma}{(\eta+\lambda \psi-1)+(\lambda \delta-\gamma)}\right]^{1 / 1-\alpha} \cdot\left[\frac{L^{\beta}(1-t)}{d}\right]^{\alpha / 1-\alpha} \cdot \beta L^{\beta}(1-t)\right\} \\
& \cdot\left[\frac{\lambda \delta-\gamma}{(\eta+\lambda \psi-1)+(\lambda \delta-\gamma)}\right]^{\alpha / 1-\alpha} \cdot \frac{\lambda(\lambda \delta-\gamma)}{[(\eta+\lambda \psi-1)+(\lambda \delta-\gamma)]^{2}} \\
& -\lambda \cdot \ln \left[\frac{\eta+\lambda \psi-1}{\lambda \delta-\gamma}\right]-(\eta+\lambda \psi-1) \cdot \frac{1}{\frac{\eta+\lambda \psi-1}{\lambda \delta-\gamma} \cdot \frac{\lambda}{\lambda \delta-\gamma}}
\end{aligned}
$$

3. Detailed differentiation with respect to $\delta$ :

$$
\begin{aligned}
\frac{\partial(U-D)}{\partial \delta}= & -\lambda \cdot \ln \left\{\left[\frac{\lambda \delta-\gamma}{(\eta+\lambda \psi-1)+(\lambda \delta-\gamma)}\right]^{1 / 1-\alpha} \cdot\left[\frac{L^{\beta}(1-t)}{d}\right]^{\alpha / 1-\alpha} \cdot \beta L^{\beta}(1-t)\right\} \\
& -\frac{1}{1-\alpha} \cdot[(\eta+\lambda \psi-1)+(\lambda \delta-\gamma)] \cdot\left[\frac{L^{\beta}(1-t)}{d}\right]^{\alpha / 1-\alpha} \cdot \beta L^{\beta}(1-t) \\
& \left\{\left[\frac{\lambda \delta-\gamma}{(\eta+\lambda \psi-1)+(\lambda \delta-\gamma)}\right]^{1 / 1-\alpha} \cdot\left[\frac{L^{\beta}(1-t)}{d}\right]^{\alpha / 1-\alpha} \cdot \beta L^{\beta}(1-t)\right\} \\
& \cdot\left[\frac{\lambda \delta-\gamma}{(\eta+\lambda \psi-1)+(\lambda \delta-\gamma)}\right]^{\alpha / 1-\alpha} \cdot \frac{\lambda(\eta+\lambda \psi-1)}{[(\eta+\lambda \psi-1)+(\lambda \delta-\gamma)]^{2}} \\
& +(\eta+\lambda \psi-1) \cdot \frac{1}{\frac{\eta+\lambda \psi-1}{\lambda \delta-\gamma}} \cdot \frac{\lambda(\eta+\lambda \psi-1)}{(\lambda \delta-\gamma)^{2}}-\lambda \cdot \ln (1+r)
\end{aligned}
$$




\section{Appendix 2}

Let us assume that the utility function of each agent in the economy is $U=\ln \left(C_{0 t}\right)+\gamma \ln \left(C_{1 t}\right)$

The agent is trying to maximize the utility:

$$
\begin{array}{ll}
\text { Max } & \ln \left(C_{0 t}\right)+\gamma \ln \left(C_{1 t}\right) \\
\text { s.t } & C_{0 t}^{i}+\frac{C_{1 t}^{i}}{1+r}=W_{i t}(1-t)
\end{array}
$$

We get

$$
\begin{aligned}
C_{0} & =\frac{1}{1+\gamma} \cdot W(1-t) \\
C_{1} & =\frac{1+r}{1+\gamma} \cdot W(1-t) \\
S & =\frac{\gamma}{1+\gamma} \cdot W(1-t)
\end{aligned}
$$

\section{Production}

The output produced by the firm at time $t$ is:

$$
Y_{t}=K_{t}^{\alpha} L_{t}^{\beta}
$$

\section{Income distribution}

The product, $Y_{t}$, in the firm is divided between the firm owner and the workers. If labor is perfectly mobile, then workers get their marginal product, so that:

$$
W_{t}=\beta K_{t}^{\alpha} L_{1 t}^{(\beta-1)}
$$

where $W_{t}$ is the wage of a worker.

In each firm we get that: $W_{t} L_{t}=\left(\beta K^{\alpha} L_{t}^{(\beta-1)}\right) L_{t}=\beta Y_{t}$ is the total income of the workers.

The income of the firm owners is: $W_{p, t}=K_{t}^{\alpha} L_{t}^{\beta}-\left(\beta K^{\alpha} L_{t}^{(\beta-1)}\right) L_{t}=(1-\beta) Y_{t}$

Aggregate savings

Assuming that the number of workers is $N$ and that there is 1 firm owner, the aggregate savings in the economy would be:

$$
S_{t}=\sum_{i=1}^{N+1} S_{t}^{i}=\frac{\gamma}{1+\gamma} \cdot \sum_{i=1}^{N+1} W_{t}^{i}(1-t)=\frac{\gamma}{1+\gamma} \cdot Y_{t}(1-t)
$$

\section{Equilibrium}

The amount of capital in the next generation would be $K_{t+1}=(1-d) K_{t}+I_{t}$ and the net investment would be financed by the savings of the current generation, so that $S_{t}=I_{t}$. We would get that:

$$
K_{t+1}=(1-d) K_{t}+S_{t}
$$

Substituting (A.10) in (A.11) we get:

$$
K_{t+1}=(1-d) K_{t}+\frac{\gamma}{1+\gamma} \cdot Y_{t}(1-t)
$$

Substituting the production function (A.8) into (A.12) we get:

$$
K_{t+1}=(1-d) K_{t}+\frac{\gamma}{1+\gamma} \cdot K_{t}^{\alpha} L_{t}^{\beta}(1-t)
$$

Given that population size is fixed, we get that the capital stock of the next generation is determined by the current stock of capital.

Stationary equilibrium will exist for $K_{t+1}=K_{t}$ and we get: 


$$
K_{t}=(1-d) K_{t}+\frac{\gamma}{1+\gamma} \cdot K_{t}^{\alpha} L_{t}^{\beta}(1-t)
$$

or

$$
K_{t}=\left[\frac{\gamma}{1+\gamma} \cdot \frac{L_{t}^{\beta}(1-t)}{d}\right]^{1 / 1-\alpha}
$$

Substituting the in production function (A.8) we get:

$$
Y_{t}=\left[\frac{\gamma}{1+\gamma} \cdot \frac{L_{t}^{\beta}(1-t)}{d}\right]^{\alpha / 1-\alpha} \cdot L_{t}^{\beta}
$$

or

$$
Y_{t}=\left[\frac{\gamma}{1+\gamma} \cdot \frac{1-t}{d}\right]^{\alpha / 1-\alpha} \cdot L_{t}^{\beta / 1-\alpha}
$$

